Originalien

Med Klin Intensivmed Notfmed 2021 · 116:322-331 https://doi.org/10.1007/s00063-020-00663-6 Eingegangen: 8. September 2019

Überarbeitet: 7. Dezember 2019

Angenommen: 31. Dezember 2019

Online publiziert: 18 . Februar 2020

(c) Der/die Autor(en) 2020

\section{Redaktion}

M. Buerke, Siegen

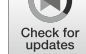

J. S. Radtke' $\cdot$ J. Götz ${ }^{2} \cdot$ S. Gielen ${ }^{2}$ F. Fischer ${ }^{1}$

'Fakultät für Gesundheitswissenschaften, Universität Bielefeld, Bielefeld, Deutschland

${ }^{2}$ Klinikum Lippe-Detmold, Detmold, Deutschland

\title{
Bettenkapazitätsanalyse für eine internistische Intensivstation
}

\section{Retrospektive Analyse und Projektion des Intensivbettenbedarfs bei über 65-Jährigen mit ausgewählten kardiovaskulären Erkrankungen}

\section{Hintergrund}

Die Intensivstation (ITS) gehört aufgrund des Einsatzes von lebenserhaltenden sowie -rettenden Maßnahmen für die Behandlung von schwerkranken Patienten zu den kostenintensivsten Bereichen im Krankenhauswesen und bedarf daher einer effizienten Steuerung $[1,8]$. Vor dem Hintergrund des demographischen Wandels mit einem Anstieg der älteren Bevölkerung wird in den kommenden Jahren ein Zuwachs des intensivmedizinischen Bettenbedarfs erwartet [12]. Zwischen 2006 und 2016 ist die gesamte Anzahl der Belegungstage auf deutschen ITS bereits um ca. $19 \%$ angestiegen [13]. Insbesondere Herz-Kreislauf-Erkrankungen stellen eine häufig auftretende Indikation bei über 65-Jährigen dar. Aufgrund der gleichzeitig vorliegenden Schwere der teilweise kritischen Erkrankungsbilder (z.B. Myokardinfarkte) sind spezielle intensivmedizinische Therapien erforderlich, sodass internistische ITS mit einem hohen Anteil an älteren Personen ausgelastet sind $[5,9]$. Somit ist eine Analyse des intensivmedizinischen Bedarfs der Patientengruppe über 65 Jahren mit kardiovaskulären Erkrankungen notwendig, um die Ressourcen auf der ITS langfristig effektiv einsetzen zu können. Weiterhin könnte sich eine unpräzise Planung und Bereitstellung von Kapazitäten einerseits lebensgefährdend auf die jeweils betroffenen Patienten auswirken und andererseits vermeidbare und hohe Kosten verursachen [1]. Dies verdeutlicht die Notwendigkeit einer effizienten Bettenkapazitätsplanung, u.a. angepasst an den demographischen Alterungsprozess, auf der ITS [11, 14]. Vergangene Studien haben sich bereits mit unterschiedlichen Methoden für eine angemessene Bettenbedarfsberechnung für ITS auseinandergesetzt. Bisher hat sich jedoch kein standardisiertes Verfahren durchgesetzt [8]. Insbesondere mathematische Modelle (z.B. Warteschlangentheorien, computerbasierte Simulationen) eignen sich für eine effiziente Bettenkapazitätsplanung. Aufgrund der Komplexität in der Umsetzung und Anwendung der teilweise sehr anspruchsvollen mathematischen Simulationsmodelle sind diese in der Praxis bislang jedoch kaum verbreitet [17].

Die vorliegende Arbeit stützt sich auf eine Empfehlung von Cochran und Roche [3], die eine simplere Abwandlung der Warteschlangentheorie mithilfe der Software Excel (Microsoft, Redmond, WA, USA) entwickelt haben, um eine barrierefreie Anwendung in der Praxis zu ermöglichen. Ziel der Arbeit ist die Analyse des retrospektiven und zukünftigen Intensivbettenbedarfs mit besonderem Fokus auf Patienten über 65 Jahre mit ausgewählten (kardiovaskulären) ICD10-Codes am Beispiel eines Maximal- versorgers in einer ländlichen Region in Nordrhein-Westfalen.

\section{Methodik}

\section{Studiendesign}

Es wurden Abrechnungsdaten der internistischen ITS eines Maximalversorgers für den Zeitraum Januar 2015 bis Dezember 2017 ausgewertet. In die Analyse wurden die 6 häufigsten Hauptdiagnosen (HD) basierend auf ICD-10-Codes der internistischen ITS miteinbezogen. Von diesen 6 Hauptdiagnosen müssen nach Aussage des Krankenhauses die folgenden zwingend in den ersten $48 \mathrm{~h}$ auf der ITS behandelt werden: I21.0 (akuter transmuraler Myokardinfarkt der Vorderwand), I21.1 (akuter transmuraler Myokardinfarkt der Hinterwand), I21.4 (akuter subendokardialer Myokardinfarkt). Außerdem sollen Patienten mit den Diagnosen I49.0 (Kammerflattern und Kammerflimmern) sowie I50.14 (Herzinsuffizienz mit Beschwerden in Ruhe) ebenfalls in den ersten $24 \mathrm{~h}$ auf der ITS behandelt werden.

\section{Datenanalyse - deskriptive Analysen}

Die deskriptiven Analysen zur Berechnung der Kapazitätsauslastungen, der Aufnahmeraten sowie der Verweildauern wurden mithilfe der Statistiksoftware 


\begin{tabular}{ll|lll}
\hline Tab. 1 Auslastung im Jahresvergleich (2015-2017) & & & \\
\hline & Tage & $\mathbf{2 0 1 5}$ & $\mathbf{2 0 1 6}$ & $\mathbf{2 0 1 7}$ \\
\hline $\begin{array}{l}\text { Unterauslastung } \\
\text { (0-69,99\%) }\end{array}$ & Anzahl & 8 & 2 & 1 \\
\hline $\begin{array}{l}\text { Ideale Auslastung } \\
\text { (70,00-80,00\%) }\end{array}$ & Anzahl & $(2,2)$ & $(0,5)$ & $(0,3)$ \\
\hline Überauslastung & $(\%)$ & $(1,6)$ & 11 & 11 \\
(80,01-100,00\%) & Anzahl & 278 & $(3,0)$ & $(3,0)$ \\
\hline $\begin{array}{l}\text { Starke Überauslastung } \\
\text { (>100,00\%) }\end{array}$ & $(\%)$ & $(78,6)$ & $(68,9)$ & 192 \\
\hline Insgesamt & Anzahl & 64 & 101 & 161 \\
& $(\%)$ & $(17,5)$ & $(27,6)$ & $(44,1)$ \\
& Anzahl & 365 & 366 & 365 \\
& $(\%)$ & $(100)$ & $(100)$ & $(100)$
\end{tabular}

zentrum (ITS) kann eine bestimmte Anzahl von Kunden (Intensivpatienten), die den Service (Intensivbehandlung) benötigen, bis $\mathrm{zu}$ einer bestimmten Anzahl simultan bedienen. Sobald die vorhandenen Kapazitäten (Intensivbetten) ausgelastet sind, muss ein neuer Kunde (Intensivpatient) warten [2].

Das Verfahren wurde in dieser Arbeit mithilfe von Microsoft Excel 2016 verwendet. Für die Berechnung des Bettenbedarfs, beruhend auf der Warteschlangentheorie, waren die Parameter Bettenkapazitäten, Verweildauer und Aufnahmeraten grundlegend. Für die Intensivpatienten wurde die berechnete Verweildauer miteinbezogen und für die Normalstationspatienten (mit einer der ausgewählten HD) ist die Annahme getroffen worden, dass diese eine durchschnittliche Verweildauer von 2 Tagen auf der ITS aufweisen würden. Aufgrund der hohen Anzahl an Patienten mit dem ICD-10-Code I50.14 auf der ITS

$V D_{i, T}:=$ Verweildauer auf Station $i \mathrm{im}$ Zeitraum $T$

$$
\begin{aligned}
& \text { Auslastung/Tag \% = } \\
& \frac{\text { Anzahl Patienten }_{\text {Mitternacht }}}{12 \text { Betten }} \times 100
\end{aligned}
$$

Die Aufnahmeraten definierten sich über die durchschnittliche Anzahl an Aufnahmen pro Tag je Monat und wurden anhand der folgenden Gl. 2 berechnet:

$$
A R_{i}=\frac{F_{i, T}}{t_{i, T}} \forall i
$$

$\mathrm{AR}_{i}:=\quad$ Aufnahmerate für Station $i$ pro Tag

$F_{i, T}:=\quad$ Fälle aufStation $i$ in Zeitraum $T$

$t_{i, T}:=\quad$ Anzahl Tage auf Station $i \mathrm{im}$ Zeitraum $T$

Um die durchschnittliche Verweildauer pro Monat zu ermitteln, wurde die Verweildauer jedes einzelnen Falls anteilig auf jeden Monat aufgeteilt. Anschließend wurde die Summe der Verweildauertage jedes Monats durch die Anzahl der Patienten in dem jeweiligen Monat dividiert, um die durchschnittliche Verweildauer

\footnotetext{
1 Im M/M/c-Warteschlangensystem sind Ankunftszeit und Bedienzeit (hier: Verweildauer) exponenziell verteilt. Die Wartenden werden von c Agenten (hier: Intensivbetten) bedient. Das Modell nimmt einen unbegrenzten Warteraum an und es gibt keine Einschränkungen hinsichtlich der Wartezeit der Kunden (hier: Intensivpatienten; [6]). Dieses Modell stellt die Basis für die Bettenbedarfsberechnung dar.
}

(-Abb. 2) und der noch bedeutsameren Patientenzahl auf der Normalstation (2015: 376 Patienten, 2016: 429 Patienten, 2017: 387 Patienten) ist davon auszugehen, dass der Einschluss dieser Patientengruppe bei der Bettenbedarfsberechnung eine Verzerrung des eigentlichen Intensivbettenbedarfs hervorrufen könnte. Da retrospektiv nicht einzuschätzen ist, inwieweit ein tatsächlicher Bedarf an Intensivplätzen bei der hohen Anzahl an Normalstationspatienten mit diesem ICD-10-Code vorlag, wurde die Bettenbedarfsberechnung sowohl für die gesamte Patientengruppe mit allen $6 \mathrm{HD}$ als auch für die Gruppe ohne Einschluss des ICD-10-Codes I50.14 durchgeführt. Der Bettenbedarf für die beiden Gruppen ist mit den folgenden Gln. 4 und 5 berechnet worden:

$$
B_{T}=\sum_{i} A R_{i, T} * V D_{i, T}
$$

$B_{T}:=\quad$ Bettenbedarf für Zeitraum $\mathrm{T}$ (bei einer Zielauslastung von $100 \%)$

$\mathrm{AR}_{i, T}:=$ Aufnahmerate für Station $i$ pro Tag für Zeitraum $T$ 
Med Klin Intensivmed Notfmed 2021 • 116:322-331 https://doi.org/10.1007/s00063-020-00663-6

(c) Der/die Autor(en) 2020

\section{J. S. Radtke · J. Götz · S. Gielen · F. Fischer}

\section{Bettenkapazitätsanalyse für eine internistische Intensivstation. Retrospektive Analyse und Projektion des Intensivbettenbedarfs bei über 65-Jährigen mit ausgewählten kardiovaskulären Erkrankungen}

\section{Zusammenfassung}

Hintergrund. Der Anstieg der älteren Bevölkerungsgruppe und der damit einhergehende Zuwachs des intensivmedizinischen Bedarfs betont die Notwendigkeit einer effizienten Bettenkapazitätsanalyse. Insbesondere Herz-Kreislauf-Erkrankungen stellen ein häufig auftretendes Erkrankungsbild bei über 65-Jährigen dar. Ziel dieser Arbeit war somit die Analyse des retrospektiven und zukünftigen Intensivbedarfs von älteren Patienten über 65 Jahre mit 6 ausgewählten (kardiovaskulären) Codes der Internationalen statistischen Klassifikation der Krankheiten und verwandter Gesundheitsprobleme (ICD-10) am Beispiel einer Einrichtung der Maximalversorgung in einer ländlichen Region.

Methodik. Für die retrospektive Analyse wurden Daten für den Zeitraum 2015-2017 deskriptiv und bivariat ausgewertet. Die Analyse des Intensivbettenbedarfs erfolgte anhand der Warteschlangentheorie. Ergebnisse. In dem betrachteten Zeitraum lagen die monatlichen Auslastungsraten kontinuierlich über der idealen Auslastungsrate von $80 \%$ und zum Teil sogar über $100 \%$. Insbesondere die Nachfrage von Patienten mit I50.14 war im gesamten Krankenhaus sehr hoch. Die Bettenbedarfsanalyse zeigt einen Anstieg von 9 benötigten Betten im Jahr 2017 auf 11 Betten bis zum Jahr 2030 für die 6 Diagnosegruppen. Ohne Einschluss der Diagnosegruppe I50.14 wird sowohl retrospektiv als auch zukünftig etwa die Hälfte der Betten, die bei Einschluss aller 6 Diagnosen benötigt wurden, nachgefragt. Diskussion. Der Effekt des demographischen Wandels auf den Intensivbettenbedarf ist bereits heute sichtbar. Zudem zeigen die Analysen, dass ein weiterer Anstieg des Bedarfs in Zukunft zu erwarten ist. Die Ergebnisse bestätigen die Notwendigkeit einer an den Bedarf angepassten Intensivkapazitätsplanung. Vor Erweiterung der Bettenkapazitäten wäre jedoch die Analyse von Kriterien, die eine intensivmedizinische Behandlung bedingen, notwendig, um primär Kapazitäten für Patienten mit einem realen Intensivbedarf vorzuhalten.

Schlüsselwörter

Intensivmedizin · Demographische Alterung · Kardiovaskuläre Diagnosen · Intensivbettenplanung $\cdot$ Warteschlangentheorie

\section{Bed capacity analysis for an intensive care unit. Retrospective analysis and projection for need of intensive care beds in over 65 year olds with selected cardiovascular diseases}

\section{Abstract}

Background. The increasing number of elderly individuals in the population and the simultaneous increase of the intensive care demand emphasizes the relevance of an efficient bed capacity analysis. Particularly, cardiovascular diseases represent a frequently occurring disease in the population group over 65 years of age. The objective of the following paper is the analysis of the retrospective and prospective intensive care demand by patients over 65 years with 6 selected (cardiovascular) codes of the International Statistical Classification of Diseases and Related Health Problems (ICD-10).

Methods. For the retrospective analysis, data from 2015-2017 were analyzed applying descriptive and bivariate methods. The analysis of the intensive care bed demand was based on the queuing theory.

Results. The monthly capacity utilization rates were constantly higher than the target capacity utilization rate of a maximum of $80 \%$ and in some cases even higher than $100 \%$. In particular, the demand of patients with 150.14 was very high throughout the entire hospital. The bed demand analysis shows an increase from 9 needed beds in 2017 to 11 beds by 2030 for the 6 diagnosis groups. Regarding the 5 diagnosis groups without 150.14 , only approximately half of the required beds were needed, retrospectively and in future.
Conclusion. The effect of demographic change on the intensive care demand already exists, and a continuing, prospective increase of the demand is expected. The results underline the need of effective and demandoriented intensive care capacity planning. However, prior to expanding bed capacities, the analysis of admission criteria of intensive care unit patients is necessary to reserve capacities primarily for patients with real intensive care needs.

\section{Keywords}

Intensive care medicine - Demographic aging Cardiovascular diseases - Intensive care bed planning · Queuing theory
$\mathrm{VD}_{i, T}:=$ Verweildauer auf Station $i$ in Tagen für Zeitraum $T$

$$
C=\frac{B}{\rho}
$$

$\mathrm{C}:=$ Bettenbedarf (bei einer Zielauslastung von $80 \%$ )

$\mathrm{B}:=$ Bettenbedarf (bei einer Zielauslastung von $100 \%)$

$\rho:=$ Zielauslastung $(80 \%)$
Um anschließend den Bettenbedarf für diese Patientengruppe für 2020, 2025 und $2030 \mathrm{zu}$ prognostizieren, wurde der Bettenbedarf mithilfe des prozentualen Bevölkerungsgruppenanstiegs für die 3 Zeiträume aus der Bevölkerungsvorausberechnung für die Region berechnet. Für die Prognoserechnung wurde die Annahme getroffen, dass sowohl die Verweildauer als auch die Zielauslastungsrate konstant bleibt. Für die
Zielauslastung wurden $80 \%$ festgelegt, um die Möglichkeit zu haben, jederzeit intensivpflichtige Notfälle aufnehmen und zudem kurzfristigen Personalausfall (z.B. durch Erkrankungen) auffangen zu können.

Die Bedarfsberechnung für 2016 diente als Basis für die Hochrechnungen, da für 2017 noch keine Informationen über die Bevölkerung (und insbesondere den Bevölkerungsanteil der über 


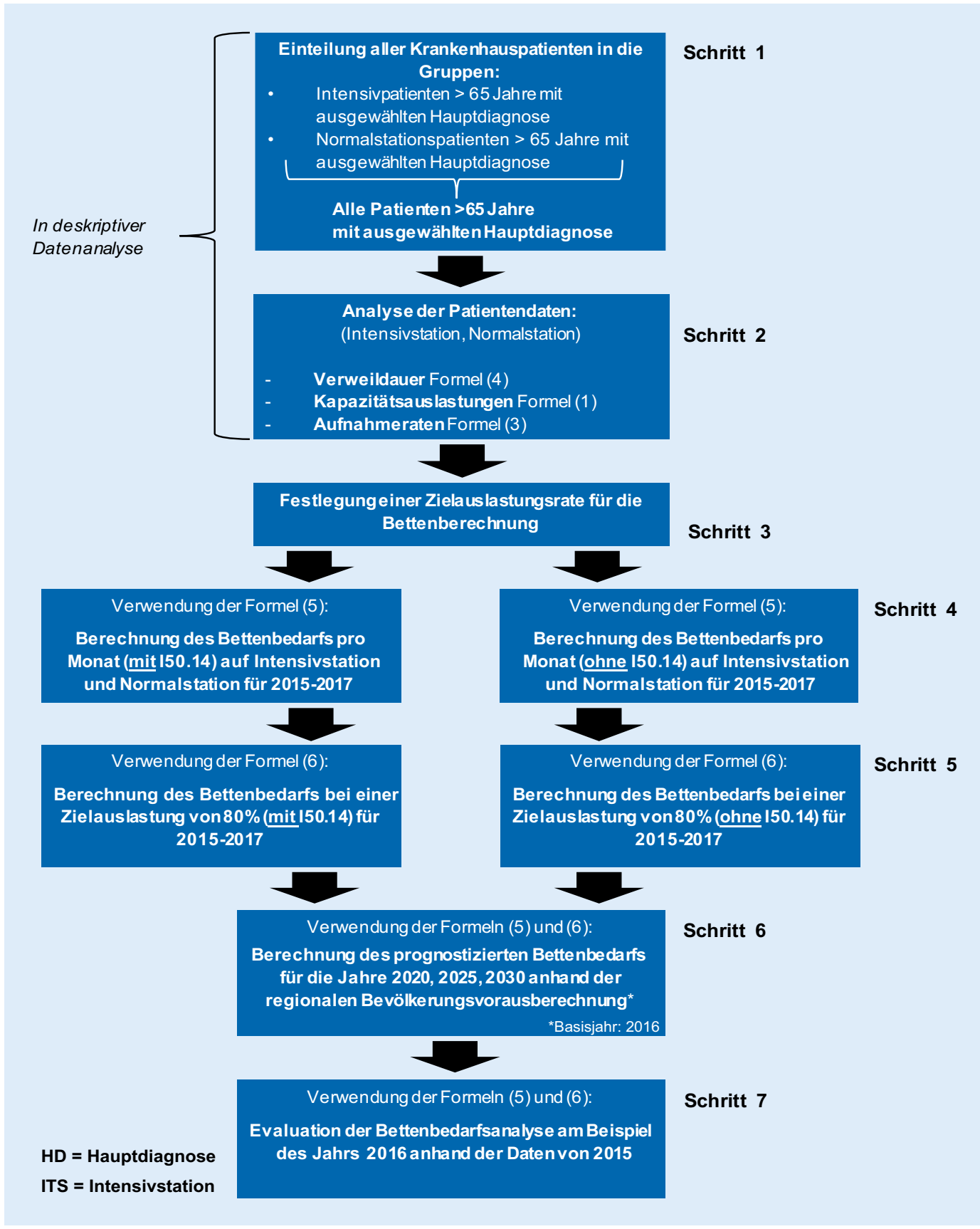

Abb. $1<$ Vorgehensweise der Bettenbedarfsanalyse

65-Jährigen) in der Region zur Verfügung gestanden haben. Zunächst war die Berechnung der Aufnahmerate für diese Patientengruppe in den Jahren 2020, 2025 und 2030 wichtig, bevor anschließend der Bettenbedarf für diese Jahre prognostiziert wurde. Für die Berechnung der zukünftigen Aufnahmerate wurde Gl. 2 entsprechend mit dem Wachstumsfaktor des jeweiligen Jahrs multipliziert. Anschließend wurde die neu berechnete Aufnahmerate in die Gln. 4 und 5 eingesetzt, um den
Bettenbedarf für das individuelle Jahr zu berechnen. Die Vorgehensweise der Bettenbedarfsanalyse ist in $\mathbf{A b b} 1 \mathrm{de}$ tailliert dargestellt.

\section{Ergebnisse}

\section{Deskriptive Analysen}

In allen 3 betrachteten Jahren (20152017) waren jeweils etwas mehr als 1000 Patienten auf der ITS. Das durchschnittliche Alter der Intensivpatienten lag im Jahr 2015 bei 66,7 Jahren, 2016 bei 67,4 Jahren und 2017 bei 66,6 Jahren. Bei ungefähr einem Drittel der Intensivpatienten in den Jahren 2015-2017 wurde eine der in dieser Studie fokussierten Hauptdiagnosen diagnostiziert. Innerhalb dieser zusammengefassten Diagnosegruppen waren ca. zwei Drittel der Patienten über 65 Jahre alt. Weiterhin mussten $34 \%$ der Intensivpatienten mit den untersuchten Hauptdiagnosen beatmet werden. Die Intensivfälle mit Beatmungspflicht sind zwischen 2015 


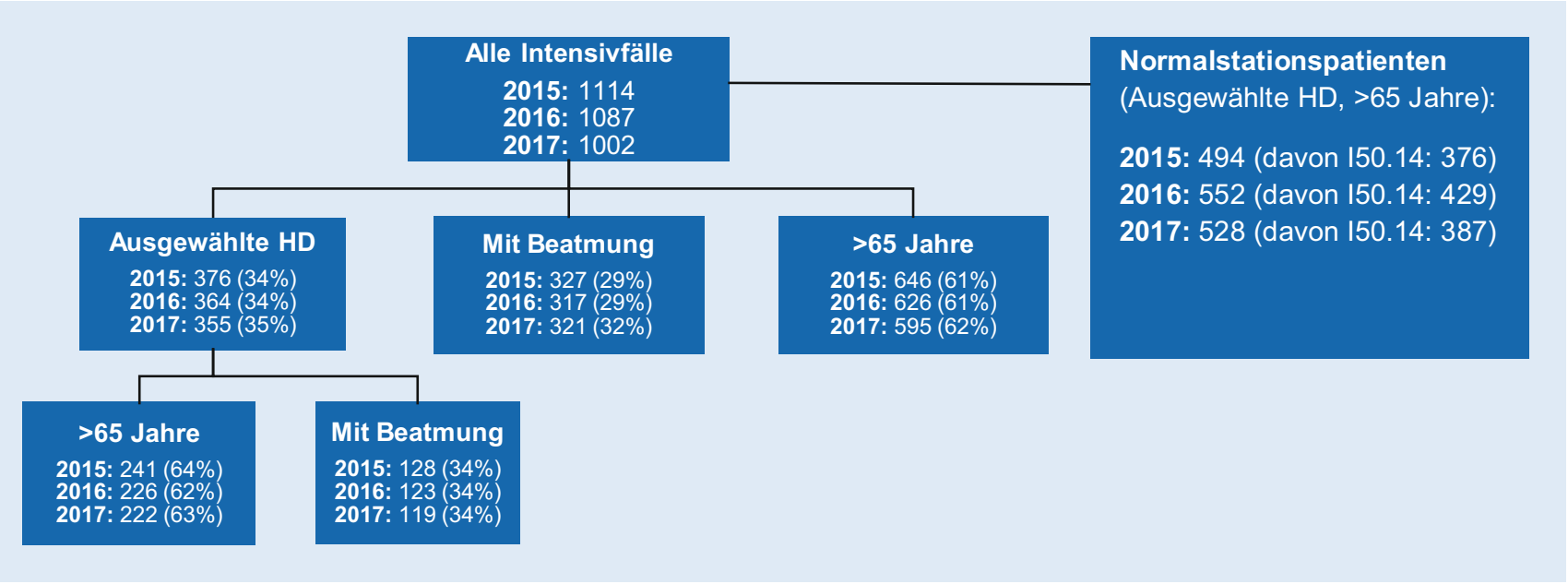

Abb. 2 A Absolute und relative Häufigkeiten der analysierten Patientengruppen auf der ITS und absolute Häufigkeiten der Normalstationspatienten > 65 Jahre mit ausgewählten HD (2015-2017)

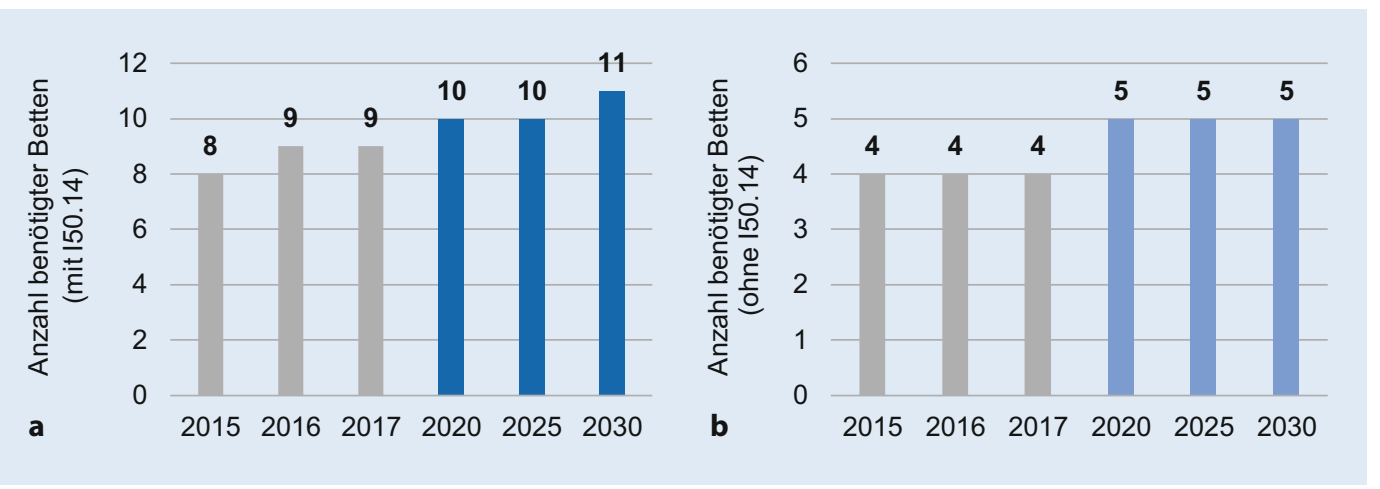

Abb. $3<$ Prognostizierter Bettenbedarf a mit 150.14 und b ohne 150.14 und 2017 von $29 \%$ auf $32 \%$ leicht angestiegen. Der Anteil der über 65-jährigen Intensivpatienten war in den 3 Jahren relativ stabil. Zusätzlichen wurde in jedem Jahr ein hoher Anteil (mind. ein Drittel der gesamten Intensivpatienten) an $\mathrm{Pa}$ tienten die älter als 65 Jahre sind und eine der ausgewählten Hauptdiagnosen hatten, lediglich auf der Normalstation behandelt (-Abb. 2).

Insgesamt zeigt sich in allen 3 betrachteten Jahren eine deutliche Überauslastung (•Tab. 1). Der Anteil der Tage mit einer Unterauslastung (unter $70 \%$ ) ist in den Jahren sehr gering und hat sich zwischen 2015 und 2017 deutlich reduziert. Obwohl sich der Anteil der Tage mit einer idealen Auslastung von 2015 (1,6\%) bis 2016 und 2017 (jeweils 3\%) fast verdoppelt hat, ist eine ideale Auslastung dennoch sehr selten. Während sich der Anteil der Tage mit einer Überauslastung zwischen 80 und $100 \%$ zwischen 2015 (78,6\%), 2016 (68,9\%) und 2017
(52,6\%) reduzierte, stieg gleichzeitig der Anteil der Tage mit einer starken Überauslastung (über $100 \%$ ) innerhalb der 3 Jahre deutlich an. Im Jahr 2017 hatten demnach mehr als doppelt so viele Tage wie im Jahr 2015 eine Auslastung von über $100 \%$ (161 vs. 64 Tage).

Anhand der monatlichen Aufnahmeraten lassen sich keine saisonalen Besonderheiten identifizieren (• Tab. 2). So weist jedes Jahr einen individuellen Verlaufder Aufnahmen auf. Die meisten Aufnahmen auf der ITS gab es für das Jahr 2015 in den Monaten Januar und Februar mit durchschnittlich je 3,8 Aufnahmen pro Tag, für das Jahr 2016 in dem Monat April (3,9 Aufnahmen pro Tag) und für das Jahr 2017 in dem Monat September (3,3 Aufnahmen pro Tag). In Bezug auf die Aufnahmeraten lässt sich feststellen, dass die meisten aufgenommenen Intensivpatienten im Durchschnitt älter als 65 Jahre waren. Weiterhin wurde im Durchschnitt täglich mindestens ein Patient mit einer der ausgewählten Hauptdiagnosen auf der ITS aufgenommen. Bei durchschnittlich 3 Gesamtaufnahmen pro Tag auf der ITS wird die Bedeutung des Anteils von Patienten dieser Diagnosegruppen somit ersichtlich. Betrachtet man weiterhin die täglichen Aufnahmeraten von Patienten über 65 Jahre mit einer der ausgewählten $\mathrm{HD}$, ergibt sich für diese Gruppe ebenfalls ein bedeutender Anteil an der gesamten Intensivpatientenzahl. Die durchschnittlichen Aufnahmeraten von Beatmungspatienten sind für die Jahre 2015, 2016 und 2017 identisch. Demnach wurde durchschnittlich ein Patient mit Beatmungspflicht täglich auf der ITS aufgenommen.

Die durchschnittliche Verweildauer pro Patient ist zwischen 2015 und 2017 um ca. $10 \%$ angestiegen: Im Jahr 2015 lagen Patienten im Durchschnitt 4,2 Tage auf der ITS, im Jahr 2016 waren es 4,3 Tage und 2017 waren es 4,6 Tage. In allen 3 Jahren ist erkennbar, dass Be- 


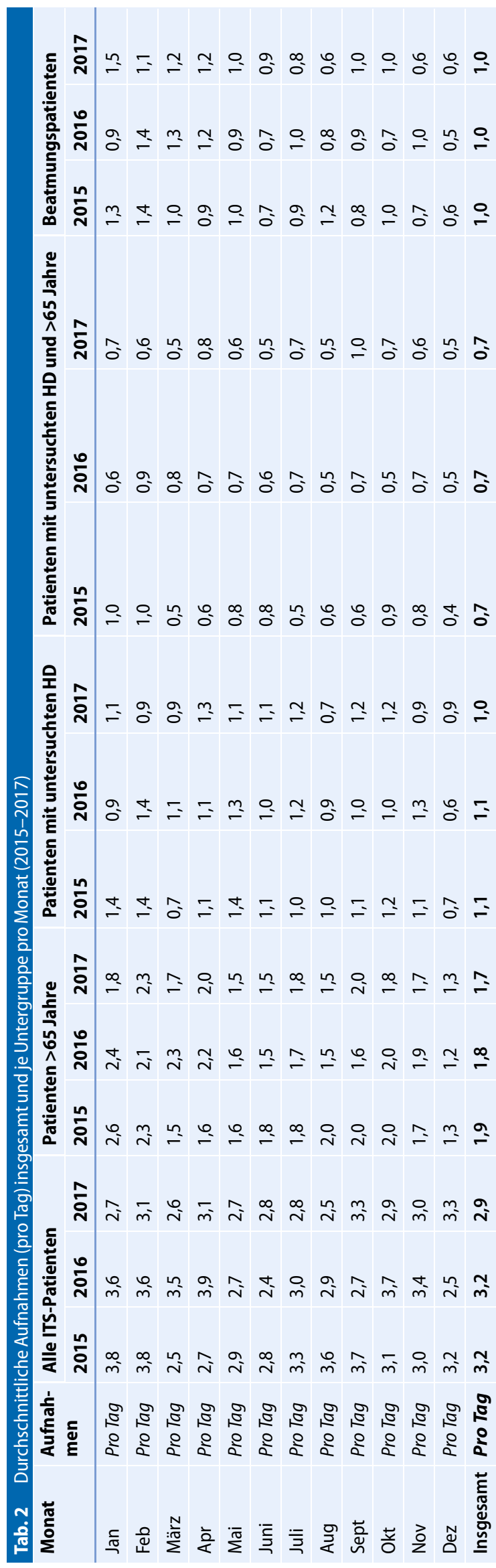

atmungspatienten im Durchschnitt am längsten (2015: 7,2 Tage; 2016: 7,0 Tage; 2017: 7,5 Tage) auf der ITS lagen. Die anderen 3 Untergruppen zeigten hingegen relativ ähnliche Verweildauern zwischen 4 und 5 Tagen auf (• Tab. 3 ).

\section{Retrospektive Bettenbedarfs- analyse für Patienten über 65 Jahre mit ausgewählten ICD-10-Codes}

In - Tab. 4 ist der Bettenbedarf für Patienten über 65 Jahre mit den ausgewählten HD für jedes Jahr dargestellt. Die Tabelle stellt den durchschnittlichen Bettenbedarf bei einer Zielauslastungsrate von $80 \%$ sowohl seitens der Patienten mit den ausgewählten $\mathrm{HD}$, die auf der ITS behandelt wurden, als auch derjenigen Patienten, die auf der Normalstation behandelt wurden, dar. Während die jeweils linken Spalten alle 6 HD inkludieren, beruhen die Werte aus den jeweils rechten Spalten auf den 5 HD ohne Einschluss von I50.14. Demnach hätte man im Jahr 2015 durchschnittlich 8 Betten $(\mathrm{I} 1+\mathrm{N} 1)$ benötigt, um der Nachfrage dieser Patientengruppe mit allen $6 \mathrm{HD}$ gerecht $\mathrm{zu}$ werden und um gleichzeitig die Zielauslastungsrate von $80 \%$ einzuhalten. Es lässt sich erkennen, dass Patienten mit der HD I50.14 mehr als die Hälfte der nachgefragten Betten (4 Betten) von allen $6 \mathrm{HD}$ (7,7 Betten) im Jahr 2015 in Anspruch nahmen. Somit wurden von den Patienten in der dargestellten Altersgruppe mit den verbliebenen HD insgesamt (aufgerundet) 4 Betten im Durchschnitt pro Tag im Jahr 2015 benötigt. Im Jahr 2016 ist ein Anstieg des Bettenbedarfs zu verzeichnen, da in diesem Jahr durchschnittlich 9 Betten pro Tag für Patienten über 65 Jahre mit den ausgewählten $6 \mathrm{HD}$ benötigt wurden. Hingegen blieb der Bettenbedarf für die analysierte Patientengruppe ohne I50.14konstant. Der durchschnittliche Bettenbedarffür die Patientengruppe (mit I50.14) änderte sich im Jahr 2017 nicht und verblieb somit bei einer unveränderten Anzahl von (aufgerundet) 9 Betten pro Tag. Ebenso blieb der Wert des Bettenbedarfs für die Gruppe ohne Berücksichtigung von I50.14 - wie in den beiden Jahren davor - mit (aufgerundet) 4 Betten konstant. 


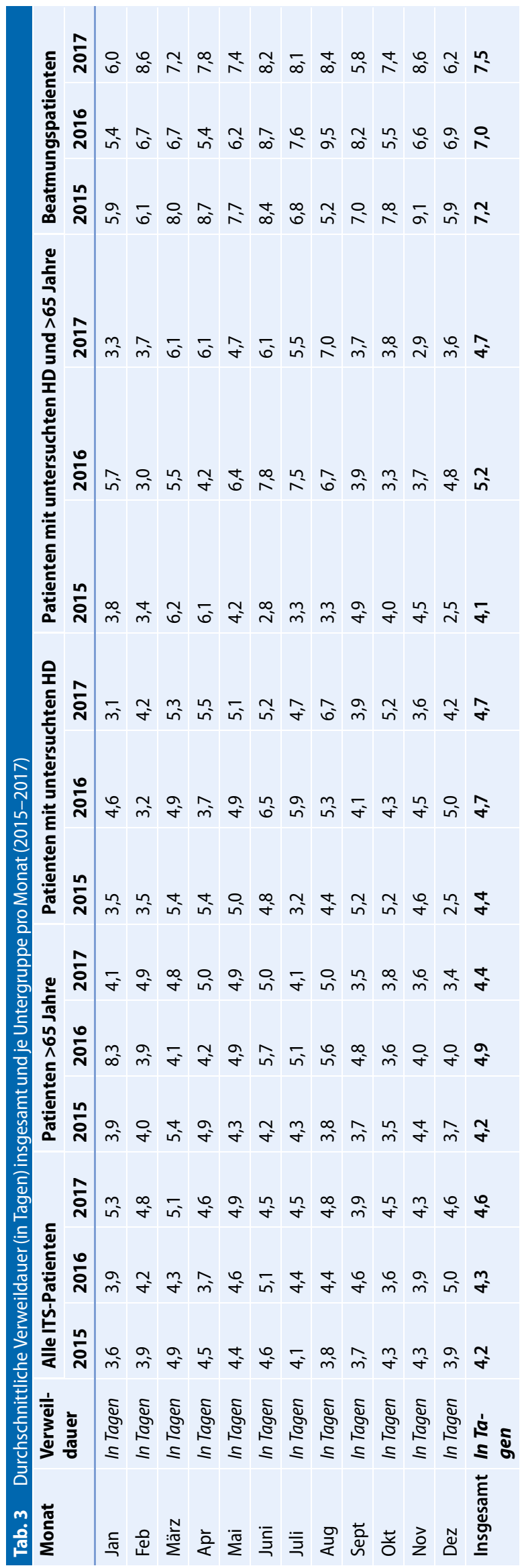

Prognostizierte Bettenbedarfsanalyse für Patienten über 65 Jahre mit ausgewählten ICD-10-Codes

Die 0 Abb. 3 bildet die prognostizierte Vorausberechnung des Bettenbedarfs einmal für die gesamten betrachteten HD-Gruppen (mit I50.14) als auch nur für die $5 \mathrm{HD}$-Gruppen (ohne I50.14) der über 65-Jährigen für die Jahre 2020, 2025 und 2030 ab. Unter Einschluss aller 6 ICD-10-Codes lässt sich erkennen, dass der Bettenbedarf bis 2030 um insgesamt 2 Betten ansteigen wird. Aufgrund des Anstiegs der älteren Bevölkerung (über 65 Jahre) in der Region um ca. 0,9\% zwischen 2016 und 2020 wird sich der Bettenbedarf um ein Bett auf (aufgerundet) $10(9,5)$ Betten bis zum Jahr 2020 erhöhen. Bis 2025 steigt der Bedarf nur geringfügig auf 9,6 Betten - trotz Wachstum der Bevölkerungsgruppe um 6,6\% - an. Aufgrund des Anstiegs der Altersgruppe über 65 Jahre in der Region um ca. $7,1 \%$, wird erwartet, dass die betrachtete Patientengruppe (über 65 Jahre mit einer der 6 HD) im Jahr 2030 voraussichtlich 11 Betten nachfragen wird (- Abb. 3a).

Bei Ausschluss des ICD-10-Codes I50.14 lässt sich eine leicht veränderte Entwicklung darstellen. Der Bettenbedarf der $5 \mathrm{HD}$ blieb für alle 3 Jahre (2015-2017) konstant bei durchschnittlich 4 Betten. Ohne Einschuss des ICD10-Codes I50.14 ist der Anstieg des Bettenbedarfs etwas langsamer ( $\mathbf{A} \mathbf{A b b} \mathbf{3 b}$ ).

Das Bevölkerungsgruppenwachstum der Einwohner über 65 Jahre in der Region $(+0,9 \%)$ zwischen 2016 und 2020 wird einen sichtbaren Einfluss auf die Bettennachfrage haben. Demnach wird im Jahr 2020 ein Bettenbedarf von aufgerundet 5 Betten (4,2 Betten) für die fokussierte Patientengruppe erwartet. Der Bedarf steigt zwar anschließend zwischen 2020 und 2025 bei einem Bevölkerungsgruppenwachstum von $6,6 \%$ noch einmal auf 4,3 Betten und anschließend bis 2030 bei einem relativen Wachstum von $7,1 \%$ auf 4,6 Betten an, jedoch wird die aufgerundete Bettenanzahl von durchschnittlich 5 nachgefragten Betten pro Patient über 65 Jahre mit einer der ausgewählten HD folglich konstant bleiben (• Abb. 3b). 


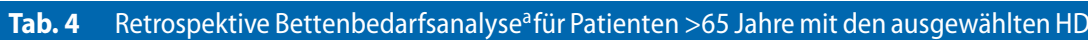
für 2015-2017

\begin{tabular}{|c|c|c|c|c|c|}
\hline \multicolumn{2}{|l|}{2015} & \multicolumn{2}{|l|}{2016} & \multicolumn{2}{|l|}{2017} \\
\hline $\begin{array}{l}\text { Bettenbedarf } \\
\text { (mit I50.14) }\end{array}$ & $\begin{array}{l}\text { Bettenbedarf } \\
\text { (ohne I50.14) }\end{array}$ & $\begin{array}{l}\text { Bettenbedarf } \\
\text { (mit I50.14) }\end{array}$ & $\begin{array}{l}\text { Bettenbedarf } \\
\text { (ohne 150.14) }\end{array}$ & $\begin{array}{l}\text { Bettenbedarf } \\
\text { (mit I50.14) }\end{array}$ & $\begin{array}{l}\text { Bettenbedarf } \\
\text { (ohne 150.14) }\end{array}$ \\
\hline $\begin{array}{l}8 \\
(7,7)\end{array}$ & $\begin{array}{l}4 \\
(3,7)\end{array}$ & $\begin{array}{l}9 \\
(8,9)\end{array}$ & $\begin{array}{l}4 \\
(4,0)\end{array}$ & $\begin{array}{l}9 \\
(8,5)\end{array}$ & $\begin{array}{l}4 \\
(4,0)\end{array}$ \\
\hline
\end{tabular}

\section{Evaluation der Praktikabilität der prognostizierten Betten- bedarfsanalyse}

Um die Praktikabilität der hier angewandten Bettenbedarfsprognose anhand des relativen Bevölkerungswachstums zu überprüfen, wurde basierend auf der Bettenbedarfsberechnung (ohne I50.14) von 2015 der Bettenbedarffür 2016 mit einem Wachstum in der Bevölkerungsgruppe der über 65-Jährigen in der Region von $1,1 \%$ hochgerechnet und mit der tatsächlichen Bettenbedarfsberechnung (ohne I50.14) von 2016 verglichen. Zusammengefasst gibt es keinen Unterschied zwischen dem realen durchschnittlichen Bettenbedarf und dem prognostizierten Bettenbedarf für das Jahr 2016. Beide Berechnungen kommen $\mathrm{zu}$ einer Anzahl von durchschnittlich 4 benötigten Betten pro Tag in dem betrachteten Jahr (Ist: 4,0/Prognose: 3,7). Vergleicht man den prognostizierten Bettenbedarf mit dem tatsächlichen Bettenbedarf pro Monat, werden ebenfalls nur geringe Abweichungen (in der Regel \pm 1 Bett) zwischen den Monaten erkennbar.

\section{Diskussion}

Die im Rahmen dieser Studie gewonnenen Ergebnisse mittels deskriptiver Analysemethoden und der angewendeten Bettenbedarfsberechnung bestätigen die Relevanz einer effizienten und bedarfsgerechten Bettenkapazitätsanalyse. Diese Studie untersuchte unter anderem, wie sich der Intensivbettenbedarf, auch hinsichtlich relevanter Patientengruppen, in dem betrachteten Zeitraum (2015-2017) in einer Einrichtung der Maximalversorgung in einer ländlichen Region darstellte.
Basierend auf der Analyse der Kapazitätsauslastungen ist festzuhalten, dass die primäre Schwierigkeit der internistischen ITS in einer Überauslastung der Intensivbetten $\mathrm{zu}$ finden ist. Obwohl das Krankenhaus die von der Deutschen Interdisziplinären Vereinigung für Intensiv- und Notfallmedizin (DIVI) und der European society of intensive medicine (ESICM) maximal empfohlene Intensivbettenanzahl von 12 Betten bereitstellt $[7,16]$, zeigt sich, dass die Nachfrage höher als das (Betten-)Angebot ist. Insbesondere die häufig auftretenden Auslastungsraten über $100 \%$, die in einer Erhöhung der Bettenanzahl resultierten, machen die Notwendigkeit einer effizienten Intensivkapazitätsplanung deutlich. Eine ideale Bettenauslastung sollte aufgrund des Arbeits- und Zeitdrucks und aufgrund der hohen Verantwortung, die das Personal einer Intensivstation $\mathrm{zu}$ tragen hat, angestrebt werden, zumal Überauslastungen zu zusätzlichen Arbeitsbelastungen beim Personal führen werden [1, 10]. Dies wiederum könnte zu negativen Folgen in Bezug auf die Patientensicherheit beitragen [1].

Bei der Analyse von zeitlichen Schwankungen in den Aufnahmeraten sowie der Verweildauer lassen sich individuelle Verläufe identifizieren. Hierbei muss berücksichtigt werden, dass die Dokumentationsqualität zu Verzerrungen führen kann und somit die jeweilige Codierung der Haupt- und Nebendiagnosen einen wesentlichen Einfluss auf die diagnoseabhängigen Auslastungsraten einnimmt. Hohe Auslastungsraten können wiederum zu fehleranfälligen Dokumentationen führen. $\mathrm{Zu}$ bedenken sind auch mögliche Fehlanreize im Abrechnungsprozess, um die Kostenintensität von ITS zu kompensieren [15]. Somit ermöglichen die Ergebnisse keine eindeutige Extrapolation dahingehend, $\mathrm{ob}$ es $\mathrm{zu}$ einer bestimmten Jahreszeit mehr oder weniger Aufnahmen gab bzw. der Auslastungsgrad unterschiedlich hoch war.

Ungefähr zwei Drittel der Patienten waren im Alter über 65 Jahre und der prozentuale Anteil hat zwischen 2015 und 2017 bereits zugenommen. Verlässt man sich weiterhin auf die Medikalisierungsthese, die besagt, dass bei steigender Lebenserwartung die Nachfrage nach medizinischen und pflegerischen Leistungen proportional zunehmen wird [4], ist auch zukünftig mit einem vermehrten Bedarf an Intensivbetten für die betrachtete Altersgruppe zu rechnen.

Aufgrund der hohen Anzahl an Fällen mit dem ICD-10-Code I50.14 auf der Normalstation wurden für jedes Jahr 2 Bettenbedarfsanalysen durchgeführt. Die beiden Analysen zeigen auf, welche Konsequenzen in der Intensivbettennachfrage entstehen würden, wenn zukünftig alle Patienten über 65 Jahre mit dem ICD-10-Code I50.14 für mindestens $48 \mathrm{~h}$ auf der ITS behandelt werden. Dementsprechend wäre die Hälfte der nachgefragten Betten seitens der 6 betrachteten HD auf die Diagnose I50.14 zurückzuführen. Konsequenterweise sollte erwogen werden, ob die Zielauslastungsrate von $80 \%$ erhöht wird, um nicht unnötige Leerkapazitäten zurückzuhalten. Dies kann jedoch aufgrund volatiler Nachfrage problematisch sein und Wartezeiten auf der ITS sollten unbedingt vermieden werden.

Im Rahmen der Bettenbedarfsprognose für 2020, 2025 und 2030 ist $\mathrm{zu}$ erwähnen, dass sich ein Anstieg in der Nachfrage zwischen jedem Jahr ereignete, dieser aber aufgrund der Aufrundung der Bettenanzahl auf ganze Betten nicht sichtbar wird. Eine Aufrundung der Bettenanzahl könnte jedoch in manchen Jahren zu einer Überschätzung des Bedarfs führen (z.B. 2020: 4,2 Betten für alle 5 Diagnosegruppen ohne I50.14). Anhand der Prognoserechnung wird der Einfluss des demographischen Wandels auf den Intensivbedarf und somit die Bedeutsamkeit von Patienten im höheren Alter erkennbar. Insofern eignet sich die hier angewandte Analyse als sinnvolle Methode, um den Intensivbedarf, jus- 
tiert an den demographischen Wandel, zu projizieren. Bedenkt man, dass die Patientengruppe der über 65-Jährigen mit einer der ausgewählten HD etwas mehr als ein Fünftel von allen Intensivpatienten ausmacht und die ITS nur über 12 Intensivbetten verfügt, ist sowohl der aktuelle als auch der zukünftige Bettenbedarf für die fokussierte Patientengruppe sehr hoch, insbesondere bei Einbezug der Diagnose I50.14. Diese Tatsache betont die Notwendigkeit, die Erkrankungsschwere bei der Aufnahme von Patienten zu ermitteln, um festzustellen, inwieweit eine Intensivpflichtigkeit tatsächlich vorliegt. Außerdem sollte vor einer Ausweitung der Bettenkapazitäten zunächst überprüft werden, inwieweit die eingesetzten Ressourcen und Behandlungsabläufe optimiert werden können, um die vorhandenen Kapazitäten effizienter zu nutzen und Ineffizienzen langfristig zu vermeiden.

\section{Limitationen}

Einige methodische Limitationen sind zu beachten. Zunächst sind die Ergebnisse nur bedingt auf andere Kliniken übertragbar, da nur ein Maximalversorger in die Untersuchung eingegangen ist. Der Bedarf an Intensivbetten ist jedoch stark von den individuellen Strukturen (z. B. hinsichtlich der Kriterien zur Aufnahme auf die ITS oder der Versorgung von I50.14) und regionalen Gegebenheiten abhängig. Um ein noch dezidierteres Bild des Intensivbedarfs älterer Patienten abbilden zu können, sind zudem noch weitere wesentliche Diagnosen, wie z.B. Infektionen und Pneumonien, in die Kapazitätsanalyse einzubeziehen. Das Forschungsinteresse dieser Arbeit zielte aber auf die genannten kardiovaskulären Hauptdiagnosen. Weitere Diagnosen sollten somit in zukünftigen Studien Beachtung finden.

Die prospektive Bedarfsplanung basiert auf der Annahme, dass lediglich Veränderungen in der Altersstruktur zu berücksichtigen sind. Der Bedarf kann sich jedoch auch bedingt durch weitere Faktoren, insbesondere durch den epidemiologischen Wandel und medizinischtechnischen Fortschritt, verändern.

\section{Fazit für die Praxis}

- Die dargestellte ITS weist sehr hohe Auslastungsraten (teilweise über $100 \%$ ) auf und bedarf daher einer Umstrukturierung der Intensivbettenplanung.

- Die Berechnung des Intensivbettenbedarfs unterliegt einer schwierigen Herausforderung. Die für die Arbeit herausgearbeitete Methodik, basierend auf der Warteschlangentheorie, hat sich jedoch als effektiv und praktikabel bewährt.

- Die Studie bestätigt die Relevanz der Intensivpatientengruppe über 65 Jahre mit kardiovaskulären Erkrankungen. Ein zukünftiger Effekt des demographischen Wandels auf den ansteigenden intensivmedizinischen Bedarf dieser Patientengruppe konnte abgeleitet werden.

- Vor Erweiterung der Intensivkapazitäten sollten standardisierte Aufnahmekriterien für die ITS bestimmt werden. Die Aufnahme aller Intensivpatienten mit einer bestimmten Diagnose - unabhängig von der Erkrankungsschwere - (z. B. 150.14) würde eine viel zu große und nicht realisierbare Bettenanzahl auf der ITS voraussetzen.

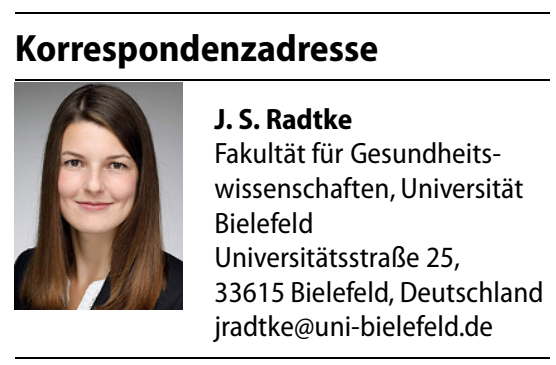

Funding. Open Access funding provided by Projekt DEAL.

\section{Einhaltung ethischer Richtlinien}

Interessenkonflikt. J.S. Radtke, J. Götz, S. Gielen und F. Fischer geben an, dass kein Interessenkonflikt besteht.

Für diesen Beitrag wurden von den Autoren keine Studien an Menschen oder Tieren durchgeführt. Für die aufgeführten Studien gelten die jeweils dort angegebenen ethischen Richtlinien.

Open Access. Dieser Artikel wird unter der Creative Commons Namensnennung 4.0 International Lizenz veröffentlicht, welche die Nutzung, Vervielfältigung, Bearbeitung, Verbreitung und Wiedergabe in jeglichem Medium und Format erlaubt, sofern Sie den/die ursprünglichen Autor(en) und die Quelle ordnungsgemäß nennen, einen Link zur Creative Commons Lizenz beifügen und angeben, ob Änderungen vorgenommen wurden.

Die in diesem Artikel enthaltenen Bilder und sonstiges Drittmaterial unterliegen ebenfalls der genannten Creative Commons Lizenz, sofern sich aus der Abbildungslegende nichts anderes ergibt. Sofern das betreffende Material nicht unter der genannten Creative Commons Lizenz steht und die betreffende Handlung nicht nach gesetzlichen Vorschriften erlaubt ist, ist für die oben aufgeführten Weiterverwendungen des $\mathrm{Ma}$ terials die Einwilligung des jeweiligen Rechteinhabers einzuholen.

Weitere Details zur Lizenz entnehmen Sie bitte der Lizenzinformation auf http://creativecommons.org/ licenses/by/4.0/deed.de.

\section{Literatur}

1. Bai J, Fügener A, Schoenfelder J, Brunner J (2018) Operations research in intensive care unit management: a literature review. Health Care Manag Sci 21:1-24

2. Barado J, Guergue J, Esparza L, Azcarate C, Mallor F, Ochoa S (2012) A mathematical model for simulating daily bed occupancy in an intensive care unit. Crit Care Med 40:1098-1104

3. Cochran J, Roche K (2008) A queuing-based decision support methodology to estimate hospital inpatient bed demand. J Oper Res Soc 59:1471-1482

4. Heigl A (2004) Älter gleich kränker? In: Scholz R, Flöthmann J (Hrsg) Lebenserwartung und Mortalität. Bundesinstitut für Bevölkerungsforschung, Wiesbaden, $\$ 41-49$

5. Heinz G, Geppert A (2013) Spezielle kardiologische Probleme auf der Intensivstation. Med Klein Intensivmed Notfallmed 108:548-548

6. Herzog A (2017) Callcenter - Analyse und Management: Modellierung und Optimierung mit Warteschlangensystemen. Springer, Wiesbaden

7. Jorch G, Kluge S, König F, Markewitz K, Notz K, Parvu V, Quintel M, Schneider D, Sybrecht GW, Waydhas C (2010) Empfehlungen zur Struktur und Ausstattung von Intensivstationen. https:// www.divi.de/empfehlungen/publikationen/ intensivmedizin/399-empfehlungen-zurstruktur-von-intensivstationen-langversion/ fil.Zugegriffen: 10. Apr. 2019

8. Luce JM, Rubenfeld GD (2002) Can health care costs be reduced by limiting intensive care at the end of life? Am J Respir Crit Care Med 165:750-754

9. Lüngen B, Zluhan M (2017) Strategisches Krankenhausmanagement in der Praxis. In: Debatin JF, Ekkernkamp A, Schulte B (Hrsg) Krankenhausmanagement, 3. Aufl. MWV Medizinisch Wissenschaftliche Verlagsgesellschaft, Berlin, S135-146

10. Meraner V, Sperner-Unterweger B (2016) Patienten, Ärzte und Pflegepersonal auf Intensivstationen. Nervenarzt 87:264-268

11. Nguyen J, Six P, Parisot R, Antonioli D, Nicolas F, Lombrail P (2003) A universal method for determining intensive care unit bed requirements. Intensive Care Med 29:849-852

12. Rubenfeld GD, Rhodes A (2014) How many intensive care beds are enough? Intensive Care Med 40:451-452 
13. Statistisches Bundesamt (2017) Intensivmedizinische Versorgung in Krankenhäusern (Betten) sowie Aufenthalte (Behandlungsfälle und Berechnungs-/Belegungstage). Gliederungsmerkmale. http://www.gbe-bund.de/. Zugegriffen: 10. Apr. 2019 (Suchverlauf: Gesundheitsversorgung $>$ Beschäftigte und Einrichtungen der Gesundheitsversorgung \Krankenhäuser > Tabelle (gestaltbar): Intensiv-medizinische Versorgung in Krankenhäusern u. a. nach Krankenhausmerkmalen)

14. Tierney L, Conroy K (2014) Optimal occupancy in the ICU: a literature review. Aust Crit Care 27:77-84

15. Thomas D, Reifferscheid A, Walendzik A, Wasem J, Pomorin N (2014) Patientengefährdung durch Fehlanreize. In: Klauber J, Geraedts M, Friedrich J, Wasem J (Hrsg) Krankenhausreport 2014. Schattauer, Stuttgart, S13-23

16. Valentin A, Ferdinande P (2011) Recommendations on basic requirements forintensive care units: structural and organizational aspects. Intensive Care Med 37:1575-1587

17. Wild C (2002) Evidenzbasierte Bedarfsplanung für Intensivbetten. Ein Assessment. Österreichische Akademie der Wissenschaften, Wien

Hier steht eine Anzeige. Springer 